and MMP13 in rat chondrocytes after IL1 $\beta$ stimulation. In vitro anti-inflammatory effect was determined by measuring the secretion of IL6, IL8, IL1 $\beta$, TNFa and IFN $\gamma$ by human monocytes. In vivo, the pharmacodynamic of our small molecules was assessed after intravenous and oral administration. Therapeutic efficacy of a compound was then evaluated in a collagen-induced arthritis model in DBA $1 / \mathrm{J}$ mice. In this model, measures of the arthritis index score, body weight, plasma level of TNFa, IL6, IL8 and IL1 $\beta$ and histological evaluation of cartilage erosion were performed.

Results: Our new series of small molecules are potent PAR2 inhibitors (IC50<1nM in calcium assay) with some selectivity over PAR1 and PAR4. Our compounds significantly inhibited PAR2 mediated recruitment of $\mathrm{Gz}, \mathrm{Gq}, \mathrm{G} 13$, G14 and G15. However, surprisingly, these small molecules had no effect on B arrestin 2, EPAC, Gi2 and GoB demonstrating that they are biased inhibitors. The effect of our compounds on PAR2 signalling was clearly different from 3 already existing PAR2 inhibitors described in the literature (I-117, AZ3451 and P2pal-18s). We compared the in vitro anti-hypertrophic effect on chondrocyte and anti-inflammatory effect on monocytes of these compounds to determine the importance of PAR2 signalling pathways in these cellular functions. In vivo, our small molecules had good bioavailability after oral administration of $10 \mathrm{mg} / \mathrm{kg}$ in mice (clearance $=$ $0.038 \mathrm{~L} / \mathrm{h} / \mathrm{kg} ; \mathrm{T} 1 \frac{1}{2}=9.9 \mathrm{~h} ; \mathrm{AUC}=162564 \mathrm{ng} \cdot \mathrm{h} / \mathrm{mL} ; \mathrm{Cmax}=9005 \mathrm{ng} / \mathrm{mL}$ ). The in vivo therapeutic efficacy of a biased PAR2 inhibitor in a model of collagen-induced arthritis will be presented.

Conclusion: Our results show the potency of biased PAR2 inhibitors to reduce both the inflammation and cartilage erosion in rheumatoid arthritis. They confirm the huge potential of PAR2 as a therapeutic target and unravel the relevance of biased antagonism of this receptor to treat rheumatic diseases.

References:

[1] McCulloch et al., Frontiers in Endocrinology, 2018; ${ }^{2}$ Hollenberg et al., British

Journal of Pharmacology, 2014; ${ }^{3}$ Sharma et al., Genes and Immunity, 2015;

${ }^{4}$ Rayees et al., Cell Reports, 2019

Disclosure of Interests: Thibaut Brugat Employee of: Domain Therapeutics, Baptiste Rugeri Employee of: Domain Therapeutics, Gaël Hommet Employee of: Domain Therapeutics, Alexia Dumont Employee of: Domain Therapeutics, Luc Baron Employee of: Domain Therapeutics, Célia Halter Employee of: Domain Therapeutics, Meriem Sémache Employee of: Domain Therapeutics, Arturo Mancini Employee of: Domain Therapeutics, Camille Amalric Employee of: Domain Therapeutics, Marie Giambelluco Employee of: Domain Therapeutics, Nathalie Lenne Employee of: Domain Therapeutics, Marjorie Sidhoum Employee of: Domain Therapeutics, Christel Franchet Employee of: Domain Therapeutics, Stanislas Mayer Employee of: Domain Therapeutics, Xavier Leroy Employee of: Domain Therapeutics, Stephan Schann Employee of: Domain Therapeutics DOI: 10.1136/annrheumdis-2020-eular.5353

\section{OP0237 THROMBOEMBOLIC SAFETY PROFILE OF TOFACITINIB AND BARICITINIB: AN ANALYSIS OF WHO VIGIBASE}

E. Vallejo-Yagüe ${ }^{1}$, S. Weiler ${ }^{1,2}$, A. M. Burden ${ }^{1} .{ }^{1}$ ETH Zürich, Department of Chemistry and Applied Biosciences, Zürich, Switzerland; ${ }^{2}$ National Poisons Information Centre, Tox Info Suisse, Associated Institute of the University of Zurich, Zurich, Switzerland

Background: The Janus Kinase (JAK) inhibitors tofacitinib and baricitinib are new targeted treatments for rheumatoid arthritis. Recent concerns regarding the risk of thrombosis have led to warnings by the European Medicines Agency (EMA) and the Food and Drug Administration (FDA) ${ }^{1,2}$.

Objectives: To examine the safety reporting of tofacitinib and baricitinib, with focus on thromboembolic events.

Methods: Individual case safety reports (ICSRs) for tofacitinib and baricitinib were retrieved from the World Health Organization (WHO) global database (VigiBase) in April 2019. The primary outcomes were deep vein thrombosis (DVT) and pulmonary thrombosis (PT) or pulmonary embolism (PE). A disproportionality analysis was conducted by estimating the reporting odds ratio (ROR) and $95 \%$ confidence intervals (Cls) to compare the observed versus expected reporting ratio of DVT or PTIPE for tofacitinib or baricitinib. The ROR were calculated worldwide and stratifying by reporting from Europe or the US. In a secondary analysis, further thrombotic-related outcomes were investigated.

Results: In both tofacitinib $(n=40,017)$ and baricitinib $(n=2,138)$ ICSRs, patients with reported DVT or PTIPE were older and had higher reporting of pro-thrombotic medications (e.g., contraceptives) or indicators of thromboembolic risk (i.e., antithrombotic treatment). The use of tofacitinib was associated with a significant increased reporting for DVT (ROR: 2.37 95\% Cl 1.23-4.56) and PTIPE (ROR $2.3895 \% \mathrm{Cl} 1.45-3.89$ ) in Europe. In the US, tofacitinib was only associated with an elevated reporting of PT (ROR: $2.05 \% \mathrm{Cl}$ 1.45-2.90). Baricitinib was associated with a 3-fold increased risk of reporting for DVT (ROR: $3.4795 \% \mathrm{Cl}$ 2.18-5.52) or PTIPE (ROR: $3.4495 \% \mathrm{Cl}$ 2.43-4.88) in Europe, which accounted for $97 \%$ of all baricitinib ICSRs. Secondary thrombotic-related outcomes were poorly reported overall in VigiBase.

Conclusion: This study supports the cautious use of JAK inhibitors in patients with rheumatoid arthritis who have a high thrombotic risk profile. Moreover, a potential class effect of JAK inhibitors cannot be ruled out.

\section{References:}

[1] FDA Drug Safety Communication. Safety trial finds risk of blood clots in the lungs and death with higher dose of tofacitinib (Xeljanz, Xeljanz XR) in rheumatoid arthritis patients; FDA to investigate. Food and Drug Administration (FDA) http://www.fda.gov/drugs/drug-safety-and-availability/ safety-trial-finds-risk-blood-clots-lungs-and-death-higher-dose-tofacitinibxeljanz-xeljanz-xr (2019).

[2] EMA confirms Xeljanz to be used with caution in patients at high risk of blood clots. EMA/608520/2019. https://www.ema.europa.eu/en/documents/ referral/xeljanz-article-20-procedure-ema-confirms-xeljanz-be-used-caution-patients-high-risk-blood-clots_en.pdf (2019).

Acknowledgments: We are thankful to every pharmacovigilance centre and contributor to the WHO Programme for International Drug Monitoring and VigiBase.

While the authors used data from the VigiBase, the WHO global database of ICSRs as a source of information, the conclusions do not represent the opinion of the Uppsala Monitoring Centre (UMC) or the WHO.

Disclosure of Interests: Enriqueta Vallejo-Yagüe Employee of: Synovo GmbH 2012-2018 (not related to this abstract), Stefan Weiler Consultant of: Gedeon-Richter for drug safety 2017 (not related to this abstract), Andrea Michelle Burden: None declared DOI: 10.1136/annrheumdis-2020-eular.2823

\begin{tabular}{|l|l}
\hline OP0238 & RISK OF HERPES ZOSTER IN PATIENTS WITH \\
RHEUMATOID ARTHRITIS UNDER BIOLOGICAL, \\
TARGETED SYNTHETIC, AND CONVENTIONAL \\
SYNTHETIC DMARD TREATMENT
\end{tabular}

A. Strangfeld $^{1}$, I. Redeker ${ }^{1}$, J. Kekow ${ }^{2}$, G. R. Burmester ${ }^{3}$, J. Braun ${ }^{4}$, A. Zink ${ }^{1,3}$. ${ }^{1}$ German Rheumatism Research Centre, Berlin, Germany; ${ }^{2}$ Scientific Advisory Board, Vogelsang-Gommern, Germany; ${ }^{3}$ Charité University Medicine, Berlin, Germany; ${ }^{4}$ Rheumatologist, Herne, Germany

Background: The risk of herpes zoster $(\mathrm{HZ})$ is higher in patients with rheumatoid arthritis (RA) than in the general population. This risk is further increased with biologic disease-modifying anti-rheumatic drugs (bDMARDs) such as tumour necrosis factor inhibitors (TNFi) and targeted synthetic (ts)DMARDs such as Janus kinase inhibitors (JAKi) compared to patients taking conventional synthetic (cs)DMARDs such as methotrexate (MTX).

Objectives: To compare incidence rates of $\mathrm{HZ}$ in RA patients under treatment with bDMARDs, tsDMARDs and csDMARDs with different modes of action and to find potential risk factors.

Methods: Data of patients enrolled in the German biologics register RABBIT from 2007 onwards with the start of a bDMARD, tsDMARD or a change in csDMARD treatment were analysed. Patients were included when at least one follow-up documentation was available. All HZ events reported until 30 April 2019 were identified and assigned to treatments administered within the 3 month period prior to the $\mathrm{HZ}$ event. Crude incidence rates (IR) of $\mathrm{HZ}$ were calculated per 1,000 patient years (py). Cox regression was applied to investigate risk factors for the occurrence of $\mathrm{HZ}$ with and without inverse probability weights (IPW) to adjus for confounding by indication.

Results: Data of 12,470 patients $(53,218$ py of observation) were included in the analysis. A total of $452 \mathrm{HZ}$ cases in 433 patients were reported, of which 52 events were serious. The crude IRs per 1,000 py are illustrated by Figure. Adjusted for age, sex, and glucocorticoid use, a significantly increased risk was observed for treatment with monoclonal TNF antibodies (hazard ratio [HR], 1.55 [95\% Cl, 1.21-2.00]), B-cell targeted therapies ( $\mathrm{HR}, 1.45$ [95\% Cl, 1.07-1.97]), and tsDMARDs (HR, 3.55 [95\% Cl, 2.33-5.41]). Treatment with soluble TNF receptors, T-cell co-stimulation modulator, and IL-6 inhibitors were not significantly associated (Table). Adjustment with IPW amplified the effect and treatment with T-cell co-stimulation modulator and IL-6 inhibitors were also significantly associated with a higher risk compared to csDMARD treatment (Table).

Conclusion: This is the first analysis in a European prospective cohort study comparing the incidence rates and risk of $\mathrm{HZ}$ in $\mathrm{RA}$ patients under treatment with six different modes of action within one cohort to cSDMARD treatment. We found a significant association between $\mathrm{HZ}$ and treatment with JAKi. Our results also confirm a higher risk for monoclonal TNF antibodies and show a similar result for the T-cell co-stimulation modulator and B-cell targeted therapies. This study clearly supports systematic $\mathrm{HZ}$ vaccination of RA patients. 
Table. Risk of herpes zoster: Results of adjusted regression analyses with and without inverse probability weights

\begin{tabular}{|c|c|c|c|c|}
\hline & \multicolumn{2}{|c|}{$\begin{array}{l}\text { Multivariate Analysis } \\
\text { without IPW }\end{array}$} & \multicolumn{2}{|c|}{$\begin{array}{l}\text { Multivariate Analysis } \\
\text { with IPW }\end{array}$} \\
\hline & $\begin{array}{l}\text { Adjusted HR } \\
(95 \% \mathrm{Cl})\end{array}$ & P Value & $\begin{array}{l}\text { Adjusted HR } \\
\quad(95 \% \mathrm{Cl})\end{array}$ & $\begin{array}{c}\mathrm{P} \\
\text { Value }\end{array}$ \\
\hline Female sex & $1.42(1.12-1.82)$ & 0.0042 & $1.21(0.96-1.53)$ & 0.1095 \\
\hline Age per 10 years & $1.23(1.13-1.33)$ & $<.0001$ & $1.31(1.2-1.43)$ & $<.0001$ \\
\hline Glucocorticoids, $5-10$ vs $0 \mathrm{mg} / \mathrm{d}$ & $1.16(0.95-1.41)$ & 0.1577 & $1.23(1-1.52)$ & 0.0501 \\
\hline $\begin{array}{l}\text { Glucocorticoids, }>10 \mathrm{vs} 0 \mathrm{mg} / \mathrm{d} \\
\text { csDMARD treatment }\end{array}$ & $\begin{array}{l}1.58(1.02-2.46) \\
\text { Reference }\end{array}$ & 0.0417 & $\begin{array}{l}1.92(1.27-2.92) \\
\text { Reference }\end{array}$ & 0.0022 \\
\hline Monoclonal TNFi antibodies & $1.55(1.20-2.00)$ & 0.0009 & $1.63(1.25-2.12)$ & 0.0003 \\
\hline Soluble TNF receptors & $1.32(0.98-1.77)$ & 0.0683 & $1.34(0.98-1.83)$ & 0.0631 \\
\hline T-cell co-stimulation modulator & $1.41(0.97-2.05)$ & 0.0746 & $1.69(1.17-2.45)$ & 0.0048 \\
\hline B-cell targeted therapies & $1.45(1.07-1.97)$ & 0.0156 & $1.66(1.19-2.3)$ & 0.0026 \\
\hline IL-6 inhibitors & $1.31(0.97-1.77)$ & 0.0737 & $1.55(1.15-2.09)$ & 0.0045 \\
\hline JAK inhibitors & $3.55(2.33-5.41)$ & $<.0001$ & $5.01(3.45-7.28)$ & $<.0001$ \\
\hline
\end{tabular}

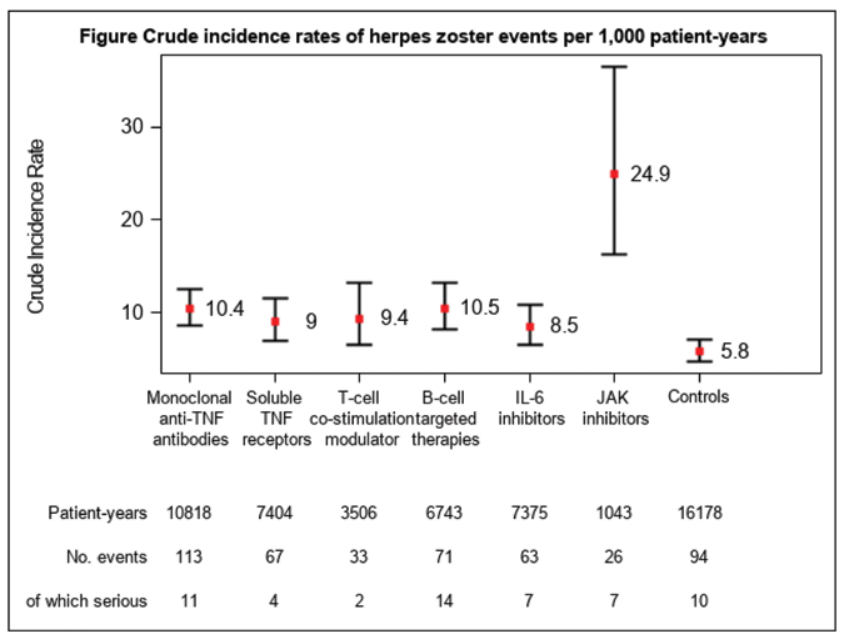

Acknowledgments: RABBIT is supported by a joint, unconditional grant from AbbVie, Amgen, BMS, Fresenius Kabi, Hexal, Lilly, MSD, Mylan, Pfizer, Roche, Samsung Bioepis, Sanofi-Aventis and UCB

Disclosure of Interests: Anja Strangfeld Speakers bureau: AbbVie, BMS, Pfizer, Roche, Sanofi-Aventis, Imke Redeker: None declared, Jörn Kekow Speakers bureau: BMS, MSD, Pfizer, Roche, Gerd Rüdiger Burmester Consultant of: AbbVie Inc, Eli Lilly, Gilead, Janssen, Merck, Roche, Pfizer, and UCB Pharma, Speakers bureau: AbbVie Inc, Eli Lilly, Gilead, Janssen, Merck, Roche, Pfizer, and UCB Pharma, Juergen Braun Grant/research support from: Abbvie (Abbott), Amgen, BMS, Boehringer, Celgene, Celltrion, Centocor, Chugai, Eli Lilly and Company, Medac, MSD (Schering Plough), Mundipharma, Novartis, Pfizer (Wyeth), Roche, Sanofi- Aventis, and UCB Pharma, Consultant of: Abbvie (Abbott), Amgen, BMS, Boehringer, Celgene, Celltrion, Centocor, Chugai, EBEWE Pharma, Eli Lilly and Company, Medac, MSD (Schering-Plough), Mundipharma, Novartis, Pfizer (Wyeth), Roche, Sanofi-Aventis, and UCB Pharma, Speakers bureau: Abbvie (Abbott), Amgen, BMS, Boehringer, Celgene, Celltrion, Centocor, Chugai, EBEWE Pharma, Eli Lilly and Company, Medac, MSD (Schering-Plough), Mundipharma, Novartis, Pfizer (Wyeth), Roche, Sanofi-Aventis, and UCB Pharma, Angela Zink Speakers bureau: AbbVie, Amgen, BMS, Gilead, Hexal, Janssen, Lilly, MSD, Pfizer, Roche, Sanofi Aventis, UCB DOI: 10.1136/annrheumdis-2020-eular.3171

\section{Preclinical models of arthritis and bone disease}

\section{OP0239 \\ WHY DOES ALCOHOL INHIBIT ARTHRITIS? - AN EXPLANATION OF THE MECHANISM OF ARTHRITIS INHIBITION BY ETHANOL}

V. Azizov ${ }^{1}$, M. V. Sokolova ${ }^{1}$, K. Sarter ${ }^{1}$, V. Temchura ${ }^{2}$, U. Steffen (Née Harre) ${ }^{1}$, M. Herrmann ${ }^{1}$, G. Schett ${ }^{1}$, M. Zaiss ${ }^{1}{ }^{1}{ }^{1}$ Department of Internal Medicine 3 , Erlangen, Germany; ${ }^{2}$ Institute of Virology, Erlangen, Germany

Background: Alcohol consumption has emerged as consistent protective factor for the development of autoimmune diseases such as rheumatoid arthritis (RA). The underlying mechanism for this tolerance-inducing effect of alcohol, however, is unknown.
Objectives: To understand the anti-arthritogenic effect of alcohol

Methods: The immune-regulatory properties of alcohol consumption in vivo were tested in the collagen-induced arthritis (CIA) and serum-induced arthritis (SIA) model as well as after immunization with T cell- dependent (NP-CGG) and independent (TNP-FICOLL) antigens. Additional experiments in vivo experiments in these models were done with acetate- the metabolite of ethanol. The models were analysed for T- cell lineage and plasma cell differentiation, germina centre formation and IgG levels and sialylation. Molecular expression of $\mathrm{T}$ follicular helper cell (TFH) activation such as IL-21, Bcl-6 and PD-1, as well as TFH: B cell conjugates were also assessed. Furthermore, TFH cells were generated in vitro, exposed to ethanol or acetate and tested for IL-21 production, PD1 expression and conjugate formation with B cells.

Results: Ethanol exposure significantly inhibited arthritis in the active adaptive immunity-driven model of arthritis $(\mathrm{CIA})$ but not in the passive innate immunity-driven model (STA) suggesting that the immune suppressive effect of alcohol is based on interference of T- and B- cell activation. In line ethanol and even more its metabolite acetate, suppressed $\mathrm{T}$ cell dependent antibody formation after NP-CGG immunization, while T cell independent antibody formation after TNP-FICOLL immunization was not suppressed. Ethanol, as well as its metabolite acetate, specifically altered the functional state of $T$ follicular helper $\left(T_{F H}\right)$ cells in vitro and in vivo, thereby exerting immune regulatory and tolerance-inducing properties. Alcohol-exposed mice showed reduced Bcl6 and PD-1 expression as well as interleukin (IL)-21 production by TFH cells, preventing proper spatial organization of TFH cells to form TFH: B cell conjugates in the germinal centre. This effect of alcohol on $T_{\text {H }}$ cells was associated with impaired autoantibody formation, higher sialylation of autoantibodies and less arthritis. In accordance, overexpression of IL-21 in vivo completely reversed the immune regulatory effects of alcohol.

Conclusion: In summary, these data provide a new mechanistic explanation for the immune regulatory and tolerance-inducing effect of alcohol consumption in arthritis.

Acknowledgments: Funden by DFG-FOR2886, DFG-CRC1181, Staedtler foundation, Johannes und Frieda Marohn-Stiftung, Else Kröner-Fresenius foundation, Interdisciplinary Centre for Clinical Research, Erlangen, BMBF-MASCARA, IMI funded project RTCure.

Disclosure of Interests: Vugar Azizov: None declared, Maria V Sokolova: None declared, Kerstin Sarter: None declared, Vladimir Temchura: None declared, Ulrike Steffen (née Harre): None declared, Martin Herrmann: None declared, Georg Schett Speakers bureau: AbbVie, BMS, Celgene, Janssen, Eli Lilly, Novartis, Roche and UCB, Mario Zaiss: None declared DOI: 10.1136/annrheumdis-2020-eular.4116

\begin{tabular}{l|l}
\hline OP0240 & A MULTIMODAL MASS SPECTROMETRY APPROACH \\
REVEALS SPECIFIC CARTILAGE MOLECULAR \\
PROFILES ASSOCIATED TO TYPE 2 DIABETIC \\
PATIENTS
\end{tabular}

M. Eveque ${ }^{1}$, P. Emans ${ }^{2}$, B. Claes ${ }^{1}$, F. Bouwman ${ }^{3}$, R. M. A. Heeren ${ }^{1}$, B. CilleroPastor ${ }^{1} .{ }^{1}$ Maastricht MultiModal Molecular Imaging (M4I) Institute, Division of Imaging Mass Spectrometry, Maastricht, Netherlands; ${ }^{2}$ Maastricht University Medical Center, Department of Orthopedic Surgery, Maastricht, Netherlands; ${ }^{3}$ Maastricht University Medical Center, Department of Human Biology, NUTRIM School of Nutrition and Translational Research in Metabolism, Maastricht Netherlands

Background: Osteoarthritis $(\mathrm{OA})$ is mainly characterized by the progressive deterioration of articular cartilage. Recent studies support that type 2 diabetes (TD2) is a risk factor to develop OA [1, 2]. However, the molecular cartilage profile of patients combining these two diseases remains unclear, and a better understanding of the different OA phenotypes should be considered for the development of personalized medicine.

Matrix-assisted laser desorption/ionization (MALDI) mass spectrometry imaging (MSI) is used to investigate the bimolecular distribution of proteins, lipids or metabolites through the in-situ analysis of tissue sections. Bottom-up proteomics focuses on the relative quantification of proteins. The combination of both technologies could be considered to reveal specific molecular profiles and help for patient classification.

Objectives: The main goal of this study is to apply a multimodal mass spectrometry approach on cartilage to reveal specific lipidomic and proteomic profiles associated to TD2 patients.

Methods: Human cartilages from OA $(n=10)$ and OA/TD2 human patients $\left(n_{b}=10\right)$ were obtained from donors undergoing total knee joint replacement. Cartilage punches of $8^{\star} 8 \mathrm{~mm}$ were sectioned at $12 \mu \mathrm{m}$ thickness for MALDI-MSI and bottom-up proteomics.

For MALDI-MSI experiments $\left(n_{a}=6 ; n_{b}=6\right)$, norharmane matrix was sprayed over the samples for the detection of lipids. Experiments were then performed in positive ion polarity at $50 \mu \mathrm{m}$ of lateral resolution using a RapifleX MALDI 